\title{
Pierre Pachet : une anthropologie du deuil
}

Pierre Pachet: An Anthropology of Bereavement

\section{Laurent Demanze}

\section{CpenEdition}

\section{Journals}

Édition électronique

URL : https://journals.openedition.org/recherchestravaux/2936

DOI : 10.4000/recherchestravaux.2936

ISSN : 1969-6434

Éditeur

UGA Éditions/Université Grenoble Alpes

Édition imprimée

ISBN : 978-2-37747-241-3

ISSN : 0151-1874

\section{Référence électronique}

Laurent Demanze, «Pierre Pachet : une anthropologie du deuil », Recherches \& Travaux [En ligne], 97 | 2020, mis en ligne le 12 novembre 2020, consulté le 02 juillet 2021. URL : http://

journals.openedition.org/recherchestravaux/2936 ; DOI : https://doi.org/10.4000/recherchestravaux. 2936

Ce document a été généré automatiquement le 2 juillet 2021.

(C) Recherches \& Travaux 


\title{
Pierre Pachet : une anthropologie du deuil
}

Pierre Pachet: An Anthropology of Bereavement

\author{
Laurent Demanze
}

1 La modernité a marqué, comme l'on sait depuis les travaux de Philippe Ariès, un basculement sans précédent dans les formes et les ritualisations du deuil ${ }^{1}$. C'est que les communautés qui enserraient le survivant de prescriptions et de pratiques rituelles ont perdu de leur pouvoir d'injonction, laissant l'endeuillé démuni, contraint de réserver l'expression de sa douleur dans le for intérieur. La mort jusqu'alors omniprésente dans la vie quotidienne, pour ainsi dire domestiquée à force d'être côtoyée, s'efface du champ social, sauf dans des espaces qui lui sont dédiés en propre. L'individu moderne est privé des cadres sociaux et des rites collectifs qui lui permettaient de symboliser l'expérience du deuil et de l'intégrer à son épaisseur de vie. Aussi est-il contraint, selon l'expression de Dominique Carlat dans Témoins de l'inactuel, «d'inventer des formes inédites de ritualisation ${ }^{2} »$.

2 La littérature est sans doute un des lieux privilégiés de cette invention: l'écrivain s'affronte à la nécessité de composer un espace de remémoration intime, un tombeau ou un monument, en quelque sorte. Mais on peut en concevoir de fragiles et de provisoires, comme a pu le faire autrefois Stéphane Mallarmé, afin d'exorciser "la solennité encombrante et définitive du monument ${ }^{3}$ ", pour édifier au contraire des tombeaux « précaires, instables, où rassembler les maigres indices, les traces fragiles " des disparus, selon les mots de Dominique Carlat ${ }^{4}$. Telles sont les œuvres de Pierre Michon ou d'Annie Ernaux, qui s'attachent à recomposer quelques silhouettes perdues, pour les célébrer modestement, en construisant quelques stèles discontinues et hypothétiques, tantôt portées par une rhétorique ironiquement emphatique, tantôt par une langue sciemment démunie.

3 Ce sont de tels monuments précaires et fragiles que compose également l'œuvre de l'essayiste Pierre Pachet, s'attachant tour à tour à la silhouette intimidante d'un père dans Autobiographie de mon père, à la perte d'une compagne dans Adieu ou au dépérissement d'une mère dans Devant ma mère 5 . L'énumération de ces trois titres dit 
l'obsession de celui qui ne cesse d'articuler l'écriture et l'expérience du deuil à une variété de dispositifs et d'élaborations formelles. D'abord une tentative de délégation de parole autobiographique, pour coïncider au plus proche avec la figure paternelle, dans un exercice d'empathie ; ensuite un journal de deuil qui s'adosse au portrait de la disparue, dans une parole qui tente de maintenir une présence et élabore «la fiction d'un échange par-delà la mort ${ }^{6} »$; enfin, une parole lucide qui se dit en face d'une mère vieillie, enfermée dans son monologue. De texte en texte, Pierre Pachet enregistre donc de manière toujours plus aiguisée l'impossible dialogue avec le disparu ou celle qui s'en va: cette trilogie du deuil prend naissance dans un désir de coïncidence des énonciations, s'approfondit dans la tension entre dialogue maintenu et nécessité d'un dessaisissement, et se clôt sur deux paroles qui ont cessé de s'ajointer l'une à l'autre, mais s'élaborent, pour ainsi dire, en parallèle. Le lecteur qui parcourt ces trois œuvres chemine ainsi de la coïncidence énonciative à une séparation radicale.

\section{Une entreprise anthropologique}

L'œuvre de Pierre Pachet circule indissolublement entre essais et écriture personnelle, selon des modes d'échange et de ressourcement. Comme le souligne Dominique Carlat, en ouverture du chapitre qu'il consacre à Adieu, "les essais se nourrissent de l'expérience d'écrivain; leur sujet est fréquemment orienté par des préoccupations liées à l'histoire subjective. Quant aux textes de création, l'ampleur de leur ambition n'apparaît pleinement qu'au regard des méditations théoriques qui constituent le fond sur lequel ils se détachent ${ }^{7}$. " Cet entrelacement inscrit toujours l'expérience la plus réservée et la plus intime sur fond de représentations collectives et d'histoire de l'individu. Devant ma mère me semble creuser cette veine pour ainsi dire anthropologique. Si j'emploie ce terme et l'accole volontiers à ce texte publié dans la collection de Jean-Bertrand Pontalis, c'est à l'invitation de Martin Rueff dans le volume de Critique qu'il consacre en 2005 à l'écrivain et qu'il introduit par un très beau texte intitulé : «Discipline des aguets ». Le critique souligne combien Pierre Pachet noue en permanence les savoirs, dans une manière de ressourcer le général sur une vigilance sans concession envers les cas singuliers et les situations du quotidien. La méthode de l'écrivain, note-t-il, "c'est fureter, affûter son regard, inventer sans cesse dans la fragilité des singularités ${ }^{8}$ ». Il y a là une "chirurgie de l'attention ${ }^{9}$ " selon la formule même de Pierre Pachet; formule qui n'est pas sans tension, tant elle accole une exigence de creuser les apparences et d'inciser les chairs, c'est-à-dire aussi de faire violence par un désir de pénétration, et en même temps d'accomplir ce geste avec une capacité d'attention ou d'hospitalité, qui est une écoute de l'autre. Violence et réparation en somme sont mêlées dans la formule oxymorique, qui dit bien sous couvert de métaphore médicale l'exigence de vigilance envers les singularités précaires.

5 Martin Rueff voit là l'émergence d'une anthropologie littéraire, qui tourne sans doute le dos à la rigueur scientifique, mais pour mieux refuser les positions de surplomb. C'est une anthropologie attentive au quotidien, curieuse de nos pratiques, hospitalière à la surface des conduites; le téléphone et la cigarette, les usages de l'automobile et les pratiques du rêve sont quelques-uns de ces territoires que l'écrivain investit pour saisir ce qui se trame au plus ordinaire de nos jours : 
L'anthropologie, ce serait donc l'orientation d'un regard (de l'écrivain, puis du lecteur qui cherche à le rejoindre au moins partiellement) sensible à la variabilité des façons d'être selon les temps, bien plus : capable de concevoir que ses propres façons de sentir sont en rapport avec des changements de la vie collective ${ }^{10}$.

6 Le souci de l'individuel va de pair avec un désir de saisir la vie collective, d'élargir une manière d'être singulière à la hauteur d'un moment historique : c'est bien là l'ambition d'une anthropologie qui ressource en permanence l'ambition de pensée à la concrétude des pratiques et qui, inversement, ouvre la pratique individuelle sur un horizon transpersonnel.

\section{Inflexions du récit de filiation : être devant}

7 Dans Encres orphelines ${ }^{11}$, j'avais souligné que les formes du récit de filiation adoptaient la temporalité de l'après-coup, du texte composé depuis le deuil, en guise d'hommage, pour reconstituer la figure du disparu, tout en endossant la posture de l'héritier. Tel est Autobiographie de mon père, où Pierre Pachet donne voix à la parole perdue du père, qui le taraude et le hante au plus intime. Mais s'il reconstitue la figure perdue, c'est pour comprendre l'énigme du deuil et se constituer à son tour : «J'avais cette voix en tête, je n'avais même qu'elle. Elle était en moi la voix la plus spontanée. [...] J'ai voulu être l'héritier ${ }^{12}$.» C'est dire que le récit de filiation se compose non seulement dans un temps d'après, mais aussi dans un souci, aporétique et impossible sans doute, de réciprocité et de symétrie.

Voilà pourquoi il faut faire un sort au titre de ce volume - Devant ma mère -, qui place Pierre Pachet non pas en position d'interlocuteur, mais de témoin : il est celui qui observe et consigne les changements d'un état, celui qui à distance ne détourne cependant pas le regard, ni ne manque d'être en présence de sa mère. Au lieu de l'horizon symétrique du récit de filiation, l'écrivain est pris «dans cette relation étrange, asymétrique» (DM, p. 7). Le titre marque en même temps la distance imposée par la maladie et la présence obstinée du fils auprès de la mère :

À présent, je ne peux plus être avec elle, ni même près ou auprès d'elle. Dans l'état où elle est, ce que je peux espérer en allant la voir et en y passant du temps, c'est qu'elle regardera dans ma direction, sans me reconnaître vraiment, et qu'elle me permettra ainsi d'être devant elle, de lui parler pour réveiller brièvement sa capacité à mimer une conversation, de lui donner à manger. (DM, p. 7)

Il y a là sans doute l'un des enjeux essentiels du récit de filiation, tel que je l'ai analysé dans Encres orphelines: non pas marquer la continuité d'une généalogie, ni dire la transmission d'un héritage symbolique, matériel ou physiologique, mais s'attacher à dire les moments de rupture et les crises de la transmission. Voilà pourquoi j'avais repris le célèbre aphorisme de René Char, "Notre héritage n'est précédé d'aucun testament", qui dit bien l'absence de norme et de repère, d'ordre symbolique, qui codifieraient les manières d'hériter.

10 Le récit de Pierre Pachet s'attache à saisir ces épisodes de méconnaissance, les marques d'étrangeté non pas dans la rupture du deuil, comme dans le récit de filiation, mais dans le présent même de la relation. Il creuse en quelque sorte l'infamiliarité qui s'installe au creux du rapport familial et dans le lien étroit entre la mère et son enfant, pour en dire les épreuves. Mais si le récit de filiation ausculte souvent les effets du temps long sur la mémoire familiale, les bouleversements de l'histoire sur les maisons et les archives - un processus de ruine, une entropie en cours -, il s'agit ici de saisir 
dans la situation présente la distance qui se creuse du fait de la vieillesse et de la maladie. C'est dans le présent même qu'un impossible dialogue se creuse, que la distance s'insinue, sans les soubresauts violents de l'histoire : deux vies, autrefois unies, se tiennent en parallèle et partent à la dérive. La coupure temporelle devient ici distance spatiale. Il faut lire en ce sens de manière malheureuse le titre de la collection de Jean-Bertrand Pontalis qui accueille le volume de Pierre Pachet, "L'un et l'autre »: le fils se tient devant la mère, et la conjonction est aussi une séparation, un écart, et désigne l'impossibilité d'un nous.

\section{Précis de décomposition}

11 Le récit de Pierre Pachet semble s'adosser aux notations précises et factuelles d'un journal : quelques dates viennent épingler un ressac de la mémoire, une intermittence du langage. L'on peut bien sûr y lire le protocole méthodologique d'une observation soigneuse, qui enregistre les étapes d'une détérioration, comme un dossier médical tenu par le fils. On peut aussi y lire l'instrument ethnographique qui permet de consigner les moments d'une immersion ou d'une observation participante, quand l'observateur est aussi un acteur social, sollicite des réponses et suscite des interactions. On peut enfin y lire les efflorescences d'un journal intime, que Pierre Pachet connaît bien, puisqu'il y a consacré un très beau volume, Les Baromètres de l'âme ${ }^{13}$ : il y dit les fonctions d'exercice mental de cette pratique de soi. Le journal serait en quelque sorte le lieu d'intersection entre un exercice intime de gouvernement de soi et le registre des exercices menés quotidiennement avec la figure maternelle, pour encourager et peut-être raviver sa capacité du langage et de l'esprit. Le journal consigne en quelque sorte simultanément l'exercice du fils et de la mère, soulignant par là l'interaction commune de la construction de soi et de la protection d'autrui.

12 Pourtant, ces dates qui émaillent le récit n'obéissent pas à une chronologie linéaire. À la manière du récit consacré aux derniers instants de son épouse, Adieu, le texte emprunte au journal pour brouiller la disposition temporelle, refuser de respecter la chronologie et distordre « l'illusion téléologique [...] coextensive au récit rétrospectif ${ }^{14}$ ». Il propose des retours arrière ou des entrelacements temporels, signe sans doute d'un dédoublement entre la notation quotidienne et l'écriture du livre, entre la ponctualité du détail enregistré et la recomposition thématique, dans un parcours de pensée. Cette division, c'est celle que l'on trouve dans la pratique ethnographique et que Vincent Debaene a bien analysée dans L'Adieu au voyage. Les deux livres de l'ethnographe ${ }^{15}$. La factualité mate n'est rien sans un travail de comparaison et d'analogie, qui ouvre le cas singulier sur un horizon universel ou anthropologique, en l'occurrence ici sur les fonctions et les fonctionnements du langage.

13 Mais la pratique désordonnée de ce journal invite aussi à y voir une manière de lutter contre un mouvement inéluctable de dégradation et de refuser une représentation de l'âge comme une trajectoire linéaire et irréversible de décomposition: "Je ne peux cependant pas limiter le thème de cet écrit à la chronique d'une perte, d'une destruction qui a lieu sous mes yeux, ou plutôt dont je constate les effets chaque fois que je lui rends visite» (DM, p. 104). Voilà pourquoi Pierre Pachet enregistre avec la sensibilité d'un sismographe les minuscules rémissions, les retours arrière ou les fragiles répits : 
Tout cela est arrivé par étapes, au cours d'une dégradation qui n'avait rien de linéaire, qui connaissait des paliers, des rémissions, des récupérations surprenantes (comme si le vaisseau spatial de sa personne s'auto-réparait provisoirement). (DM, p. 67)

Le livre est donc tendu entre deux ambitions contradictoires : faire émerger d'une part un devenir qui puisse rendre lisible les aspérités du quotidien et interroger les logiques à l'œuvre dans ce processus; contrecarrer de l'autre la linéarité d'une dégradation, marquer les inflexions surprenantes pour éviter toute lecture eschatologique de la vieillesse. Et pour cela, le journal qui consigne d'ordinaire le temps inéluctable du quotidien sera sollicité de manière désordonnée, non comme un surcroît de chaos, mais comme une lutte minime et précaire contre l'entropie.

Le livre de Pierre Pachet n'obéit donc pas aux logiques du tombeau poétique. Ce genre littéraire, fortement codifié à la Renaissance, et qui ressurgit au xixe siècle, passe comme on le sait de la célébration d'un modèle paradigmatique ou exemplaire à un hommage adressé à une figure singulière. Le tombeau devient aussi, à travers le geste de Mallarmé pour son fils Anatole, un genre qui refuse le monumental, essaye de rester en mouvement, de garder sa force de dissémination : il n'est pas une forme close, mais une forme inquiète et en mouvement, qui se cherche à tâtons, selon une succession infinie d'ajustements et de corrections. Tels sont les récits de bien des écrivains contemporains : Vies minuscules de Pierre Michon, Une femme ou La Place d'Annie Ernaux ou encore Mécanique de François Bon. Mais tel n'est pas le cas du texte de Pierre Pachet, car le tombeau, aussi ouvert à l'inachèvement soit-il, s'élabore malgré tout à partir de l'événement de la mort. Il s'agit ici davantage de l'expérience du mourir, de l'épreuve d'un processus ou d'un devenir. Ce sont ces devenirs à l'œuvre que capte le récit de Pierre Pachet, des seuils et des signes annonciateurs, des processus en cours (DM, p. 34), un désapprentissage (DM, p. 45) ou une « dé-constitution de son identité » (DM, p. 56). Le livre enregistre ces processus de déliaison du mourir, ces micro-événements qui défont les habitudes et les apprentissages, dénouent les relations. Si le récit saisit incidemment quelques aspects de la vie de la mère, des moments de son existence, il ne s'agit dès lors pas de proposer un récit rétrospectif qui tente de dire les linéaments d'une biographie ou la substance d'un individu, «tel qu'en lui-même l'éternité le change »: le récit accompagne au présent l'affrontement d'une conscience avec son propre délitement, d'un langage avec sa propre défaite.

\section{Le discours détaché}

16 Être devant la mère, c'est aussi se tenir devant elle avec le regard lucide de l'observateur, qui anatomise et ausculte. Pierre Pachet dit les aléas d'un corps et les vacillements de l'esprit de sa mère, avec la distance d'un discours détaché. Il observe «avec patience et tolérance, avec un intérêt presque scientifique, avec admiration aussi » (DM, p.17). On peut bien sûr saisir ce détachement comme une posture de protection, susceptible d'amoindrir la force du bouleversement, d'être en quelque sorte désaffectée par la dégradation progressive du corps. Il faut aussi y voir une manière d'hommage devant la résistance de la mère, qui malgré l'âge parvient encore à ramener des bribes de souvenirs, à susciter ici ou là le rire : le détachement est une manière de prendre la mesure de cette résistance, d'en dire par comparaison la force singulière. Enfin, il y a dans ce détachement une éthique de la parole, qui refuse de susciter du pathos à bon compte, sur une mère qui se meurt : «Je n'ai pas voulu que ce soit un livre 
pathétique. Je voulais la regarder avec affection mais aussi avec curiosité. Je ne voudrais pas que ce livre donne une impression poignante parce que la chose en ellemême l'est déjà suffisamment ${ }^{16}$ ", dit Pierre Pachet dans un entretien.

Celui-ci s'attache à élaborer à mesure une rhétorique de la désaffection, congédiant les marques trop pleines du pathétique ou esquivant les effusions sentimentales. C'est qu'il s'agit sans doute d'aller chercher dans les formes de la rationalité volontaire, de la maitrise conceptualisante une manière de faire écran à la violence des sentiments, de temporiser la force traumatique du deuil. Cette œuvre de Pierre Pachet me semble exemplaire d'une des forces de la tension théorique, à laquelle je suis sensible contrairement à Hélène Merlin-Kajman dans Lire dans la gueule du loup ${ }^{17}$ : le souci théorique ne me semble là ni un refoulement de l'expérience traumatique, ni une sublimation prématurée, comme le dénonce la critique, mais un travail de ressaisie de l'événement et de soi, en mobilisant les forces de l'exercice mental. C'est que, comme le souligne Dominique Carlat, Pierre Pachet ne cesse d'articuler l'une à l'autre émotion et conceptualisation; cette étroite articulation compose ainsi une œuvre à la lisière des sciences sociales et de l'intime, à la fois pour redonner une teneur subjective aux sciences sociales et inscrire l'expérience la plus intime dans un horizon collectif :

La mixité générique de ses livres semble à Pierre Pachet la conséquence nécessaire d'un double refus : d'une science de la littérature se coupant des autres domaines et reposant sur le déni de la subjectivité qui la construit; d'une création littéraire autobiographique qui se présente issue exnihilo, et comme par génération spontanée, d'une conscience de soi absolument unique ${ }^{18}$.

Ce souci de l'argumentation donne au texte l'allure d'une investigation hypothétique. Il rejoint par là la veine des récits de filiation, qui recomposent sous le signe de la supposition, du scrupule ou du conditionnel les portraits effacés des parents. Il s'agit moins dans ces récits de dresser la figure unique, que de saisir de manière diffractée et hypothétique des variations à partir d'une tache aveugle. Mais là où souvent le récit de filiation sollicite l'imagination et les médiations culturelles, de Pierre Michon convoquant L'Almanach Vermot à Gwenaëlle Aubry sollicitant le cinéma, Pierre Pachet adosse son investigation aux hypothèses les plus précautionneuses, qui marquent les zones d'ombre de l'ignorance et se détachent à peine de l'observation ${ }^{19}$. Le livre se construit comme glissement d'hypothèses, il délaisse les reconstructions imaginaires qui sont souvent des manières de transfigurer la figure perdue pour s'attacher à des tentatives de saisie rationnelle, au plus juste de ce qui a eu lieu. Saisir la mère dans sa singularité, ce n'est pas alors la refermer sur son identité, mais traquer en elle ce qu'il y a de commun et d'exemplaire, c'est élargir une réflexion "à partir du cas de ma mère " comme l'écrit Pierre Pachet. Il y va ainsi moins de l'exhibition d'une expérience intime ou de l'exposition d'une vie précaire et dégradée, que d'une manière de ressaisir la décomposition comme objet de savoir : l'ouverture du singulier au plus commun, le souci de la généralité sont une manière rationnelle et folle à la fois de rédimer la perte en un savoir, de sacrifier la douleur contre un savoir, pour renouer avec la célèbre formule tragique : pathei mathos.

Je voudrais faire que la description de ce que je constate de la détérioration de sa présence au monde ait une contrepartie positive. Comme on donne son corps à la science, je voudrais l'aider à donner à la connaissance cette détérioration même. Qu'elle puisse, que je puisse faire servir cette chronique à éclairer la constitution de la vie mentale [...]. (DM, p. 104) 


\section{Interroger les fonctions du langage}

19 Dans un bel article, intitulé "Parler des morts", Patrick Hochart rappelle que «Pierre Pachet a depuis longtemps le souci des morts, mais sans nulle morbidité ${ }^{20}$ ». Cette juste formule, le souci des morts, me semble pointer non pas les formes figées de l'hommage mais une inquiétude du langage: que signifie parler à ceux qui ne nous entendent pas? parler de ceux qui n'ont plus souci de nos mots? Que signifie, selon la saisissante formule de Pierre Pachet dans un article justement intitulé «Électre parle aux morts ${ }^{21}$ ", " parler à leur non-entendre ${ }^{22}$ " ? À travers cette interrogation l'écrivain creuse les apories du langage et de l'identité : parler aux morts, ce n'est pas sombrer dans le monde des morts, ni convoquer les revenants. C'est d'abord s'adresser aux morts devant les vivants et rappeler que la parole s'élabore à partir d'une absence ou d'un éloignement, comme une façon de tenter de résorber cette absence. S'adresser aux morts, c'est en quelque sorte dénuder un des gestes essentiels du langage: «[...] le geste mental qui nous incline vers un absent, nous dirige vers son évanouissante réalité. Sans ce geste, sans la réalité du mort, nous ne pourrions même pas nous parler

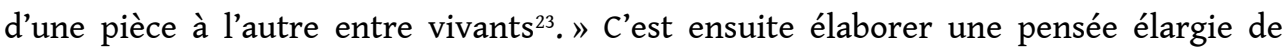
l'identité, qui prend la mesure de ces formes de délégation et d'extériorisation de soi au fil de notre histoire; parler aux morts, c'est convoquer cette part de soi absente qui dialoguait autrefois avec eux pour tenter d'y coïncider à nouveau :

Une part de notre sensibilité est avec eux, en dessous. De même que notre mémoire peut être extériorisée et enfermée hors de nous, dans un monument, dans une bibliothèque, dans un livre, de même une part de notre sensibilité peut être excentrée de nous. Pour qu'elle vive, il nous faut accepter de penser qu'elle gît quelque part, qu'elle est localisée ailleurs que dans notre corps ${ }^{24}$.

20 À travers cette impossible adresse, Pierre Pachet procède en somme à une double interrogation : un questionnement des fondements du langage et une invitation à un renouvellement de la pensée de l'individu. Cette double interrogation est au cœur de Devant ma mère, car l'écrivain retrouve dans sa situation d'interlocution les empêchements d'une parole adressée à qui ne répond pas, ne reconnaît pas. Il parle à une vivante, mais c'est pour ainsi dire retrouver dans sa situation les impératifs qu'il avait décrits dans ce "souci des morts ». Il s'agit de mettre en évidence que l'une des fonctions du langage est l'impossible adresse à autrui, la tension asymétrique qui nous porte hors de nous ${ }^{25}$.

\section{Bijoux et reliques}

21 Le livre obéit à une pratique de la notation singulière, en multipliant anecdotes et saynètes : malgré le désir de généralité, il travaille à une écriture de la scène. La scène a bien sûr une fonction exemplifiante, elle illustre un processus, dramatise dans un dialogue raté ou empêché l'évolution invisible dans les arcanes du corps. Même si la tonalité d'ensemble relève davantage d'une théâtralité de l'absurde, lorsque les mots échouent à toucher à autrui et enferment l'individu dans sa solitude, l'écriture de la scène pointe de fugaces moments d'accord; «une intimité fragile, passagère » (DM, p. 83), comme dans cette très brève séquence séparée du texte par des astérisques :

Et pourtant, le 3 décembre 2005, je suscite ceci :

Moi (en veine de confidence) : je me sens un peu seul. 
Elle : Moi aussi.

(Surprise de cet échange : laconique, ajusté, accordé. Juste.) (DM, p. 88-89) d'incongru qui pourrait distiller dans la situation une touche d'humour et dédramatiser la mort qui vient. Une telle veine est fréquente dans les récits de maladie : il s'agit de mettre en évidence les formes minimes de résistance ou de souligner les décalages salvateurs, pour faire, dans la lignée de Gilles Deleuze, de la maladie ou de l'âge les forces paradoxales d'une grande santé et d'une contestation des normes majoritaires. Pierre Pachet exclut un tel décalage, un tel renversement, par incapacité comme par refus :

Pour accomplir cette tâche, pour l'alléger, il me manque quelqu'un de comparable à Félix Guattari, jadis à la clinique de La Borde, quelqu'un qui, comme il savait le faire, relèverait la cocasserie ou l'humour - involontaires, à moitié, sans doute - de ce que dit cette démente qui m'est proche. (DM, p. 62)

Il ne s'agit pas pour l'écrivain d'alléger cette entreprise, c'est-à-dire de refuser la lucidité, ou de masquer la teneur pénible de cette observation : au contraire, la scène est ce qui réactive la distance entre la mère et le fils, ce qui lui donne son épaisseur dramatique.

L'écrivain consigne néanmoins fidèlement des points de résistance ou des rémanences de la mère d'autrefois, si exigeante et précise dans son usage des mots :

Ou encore - je chéris ces exemples, comme des bijoux qui seront aussi des reliques ce dialogue, alors qu'elle est déjà très mal en point et quasi inerte, à l'hôpital. Tu es fatiguée ? lui demandé-je en français, à la fois par compassion et pour simuler ou susciter un échange un peu normal ou digne. - Je ne suis pas fatiguée, je suis épuisée. (DM, p. 139)

La précision ou la préciosité de cet échange en fait un moment remarquable à mettre en exergue, à conserver en mémoire, comme une réminiscence précaire de sa mère. Mais ce bijou de la mémoire est aussitôt requalifié comme relique, c'est-à-dire saisi dans l'horizon de la mort de sa mère : la mort qui vient n'est jamais exorcisée par le bon mot, au contraire elle est ce qui donne à l'entreprise sa vocation mémorielle de constitution d'un trésor de rémanences à transmettre.

Aux dernières pages du livre, Pierre Pachet note : «Je suis dépositaire » (DM, p. 160) : l'écrivain réaffirme la teneur éthique d'un geste, qui consiste moins à construire un tombeau qu'à être fidèle au dynamisme de sa mère, à recueillir les indices d'une énergie et consigner les rémanences d'un désir. Il propose une manière d'anti-tombeau, qui maintienne non pas le souvenir de la morte, mais la présence vive de ce qu'elle fut jusqu'aux derniers moments. Écriture du journal et non récit rétrospectif, Devant ma mère renouvelle en ce sens la vocation éthique du récit de filiation, mais pour lui donner le tremblé du discontinu, l'incertitude du refus de la chronologie et libérer la mère d'une mort conçue comme terme du récit ou comme impulsion inaugurale. 


\section{NOTES}

1. Je renvoie évidemment à ses ouvrages essentiels : P. Ariès, Essai sur l'histoire de la mort en Occident, Paris, Seuil, 1975 et L'Homme devant la mort, Paris, Seuil, 1977.

2. D. Carlat, Témoins de l'inactuel. Quatre écrivains contemporains face au deuil, Paris, Corti, coll. « Les Essais », 2007.

3. Ibid., p. 154.

4. Ibid., p. 17.

5. P. Pachet, Autobiographie de mon père [1987], Paris, Autrement, 1994 ; Adieu, Belfort, Circé, 2001 ; Devant ma mère, Paris, Gallimard, coll. «L'un et l'autre», 2007. Les numéros de page entre parenthèses renverront à Devant ma mère, avec l'abréviation suivante : DM.

6. D. Carlat, Témoins de l'inactuel, ouvr. cité, p. 132.

7. D. Carlat, «Chapitre IV: Adieu de Pierre Pachet: portrait de l'absente, autoportrait de l'inconsolé », Témoins de l'inactuel, ouvr. cité, p. 127.

8. M. Rueff, « Discipline des aguets », Critique, $\mathrm{n}^{\circ}$ 702, 2005, p. 821.

9. Ibid., formule citée, page 821 .

10. Ibid., cité page 821.

11. L. Demanze, Encres orphelines: Pierre Bergounioux, Gérard Macé et Pierre Michon, Paris, Corti, coll. « Les Essais », 2008.

12. P. Pachet, Autobiographie de mon père, ouvr. cité, p. 7.

13. P. Pachet, Les Baromètres de l'âme. Naissance du journal intime, Paris, Hatier, 1990.

14. D. Carlat, Témoins de l'inactuel, ouvr. cité, p. 136.

15. V. Debaene, L'Adieu au voyage. L'ethnologie française entre science et littérature, Paris, Gallimard, coll. «Bibliothèque des sciences humaines », 2010.

16. "Pierre Pachet: Devant ma mère", entretien avec Pierre Pachet, 7 mai 2007, par Olivier Barrot, à la Fondation Rosa Abreu de Grancher (Cité Internationale Universitaire de Paris). Vidéo disponible sur le site de l'INA : <https://www.ina.fr/video/3344296001>.

17. H. Merlin-Kajman, Lire dans la gueule du loup. Essai sur une zone à défendre, la littérature, Paris, Gallimard, coll. «NRF essais », 2016. Sur la discussion que je mène avec cet essai, je me permets de renvoyer à mon article : L. Demanze, «Réparer et consoler : la littérature selon Hélène MerlinKajman », Romantisme : la revue du dix-neuvième siècle, Armand Colin, juin 2017.

18. D. Carlat, Témoins de l'inactuel, ouvr. cité, p. 130.

19. Par exemple : «[...] j'en suis réduit à décrire, à essayer de raccrocher les uns aux autres les faits que j'observe, ce qui est montré par sa parole et par son comportement. / Je n'ai pas le droit, vu mon ignorance de ce qui s'est passé dans son cerveau, d'émettre même des hypothèses sur les causes de ce qui l'atteint. Mais je peux essayer de reconnaître selon quelles pentes s'est effectuée cette détérioration, les formes visibles qu'elle a prises. / J'ai formulé l'hypothèse selon laquelle, à force de solitude, tout en conservant le besoin du dialogue, de la conversation, et en se mettant à jouer les divers rôles des interlocuteurs. » (DM, p. 118-119)

20. P. Hochart, « Parler des morts », Critique, $\mathrm{n}^{\circ} 712,2005$.

21. P. Pachet, «Électre parle aux morts », Nouveau Commerce, $\mathrm{n}^{\circ}$ 70, printemps 1988.

22. Ibid., p. 72.

23. Ibid., p. 73.

24. Ibid., p. 70.

25. Voir Devant ma mère, ouvr. cité, p. 153. 


\section{RÉSUMÉS}

Les livres de Pierre Pachet sont autant d'exercices d'une pensée lucide et dessillée : sur l'individu, l'amour et la vieillesse. Parmi ses centres d'attention, les figures parentales donnent lieu à deux textes saisissants: Autobiographie de mon père et Devant ma mère. Ces récits, qui oscillent entre l'intime et l'observation détachée, empruntent une exigence anthropologique et sont portés par une ambition d'interrogation large pour questionner la décomposition de l'identité, de la mémoire et du langage.

Pierre Pachet's books are all exercises of a lucid and uncluttered thought: about individuality, love and old age. Among his topics, parental figures give rise to two striking novels: Autobiographie de mon père and Devant ma mère. These narratives, oscillating between intimacy and objectivity, require an anthropological property and are driven by a large ambitious questioning about identity, memory and language decay.

\section{INDEX}

Keywords : Pierre Pachet, bereavement, anthropology, filiation, old age, identity, memory, essay Mots-clés : Pierre Pachet, deuil, filiation, anthropologie, essai

\section{AUTEUR}

\section{LAURENT DEMANZE}

Laurent Demanze est professeur à l'Université Grenoble Alpes. Ses travaux portent sur la littérature contemporaine à laquelle il a consacré des articles dans Critique, Les Temps modernes, Europe ou Études françaises. Il dirige la collection « Écritures contemporaines » aux éditions Garnier et a coordonné des collectifs, entre autres consacrés à Emmanuel Carrère, Pierre Michon ou Pierre Senges. Il est l'auteur de plusieurs essais publiés chez José Corti : Encres orphelines : Pierre Bergounioux, Gérard Macé et Pierre Michon (2008) ; Gérard Macé : l'invention de la mémoire (2009); Les Fictions encyclopédiques de Gustave Flaubert à Pierre Senges (2015) et Un nouvel âge de l'enquête (2019). 